\title{
Probing for electrical inclusions with complex spherical waves
}

\author{
TAKANORI IDE \\ Tokyo Metropolitan University \\ HIROSHI ISOZAKI \\ University of Tsukuba \\ SUSUMU NAKATA \\ Ritsumeikan University, College of Information Sciences and Engineering \\ SAMULI SILTANEN \\ Tampere University of Technology \\ AND \\ G. UHLMANN \\ University of Washington
}

\begin{abstract}
Let a physical body $\Omega$ in $\mathbb{R}^{2}$ or $\mathbb{R}^{3}$ be given. Assume that the electric conductivity distribution inside $\Omega$ consists of conductive inclusions in a known smooth background. Further, assume that a subset $\Gamma \subset \partial \Omega$ is available for boundary measurements. It is proved using hyperbolic geometry that certain information about the location of the inclusions can be exactly recovered from static electric measurements on $\Gamma$. More precisely: given a ball $B$ with center outside the convex hull of $\Omega$ and satisfying $(\bar{B} \cap \partial \Omega) \subset \Gamma$, boundary measurements on $\Gamma$ with explicitly given Dirichlet data are enough to determine whether or not $B$ intersects the inclusion. An approximate detection algorithm is introduced based on the theory. Numerical experiments in dimension two with simulated noisy data suggest that the algorithm finds the inclusion-free domain near $\Gamma$ and is robust against measurement noise. (c) 2000 Wiley Periodicals, Inc.
\end{abstract}

\section{Introduction}

\subsection{Dirichlet-to-Neumann map}

Let $\Omega$ be a bounded open set with smooth boundary in $\mathbb{R}^{d}$ with $d=2,3$, and consider the following boundary value problem:

$$
\left\{\begin{array}{rlll}
\nabla \cdot(\gamma(x) \nabla v) & = & 0 & \text { in }
\end{array}\right.
$$

Communications on Pure and Applied Mathematics, Vol. 000, 0001-0027 (2000)

(c) 2000 Wiley Periodicals, Inc. 
We assume that $\gamma \in L^{\infty}(\Omega)$ and that $\gamma(x) \geq c>0$ almost everywhere in $\Omega$. Define the Dirichlet-to-Neumann (DN) map by

$$
\Lambda:\left.f \rightarrow \gamma(x)\left(\frac{\partial v}{\partial n}\right)\right|_{\partial \Omega},
$$

where $v$ is the solution to (1.1) and $n$ is the outer unit normal to $\partial \Omega$.

The inverse conductivity problem of Calderón [12] is to determine $\gamma$ from $\Lambda$. This problem has several practical applications where $\gamma$ represents the electric conductivity inside a physical body, $v$ is the voltage potential, and $\Lambda$ models static voltage-to-current measurements on the boundary of the body. The aim of electrical impedance tomography (EIT) is to form an image of the conductivity distribution from the knowledge of $\Lambda$. See $[14,8]$ for reviews of EIT.

When $\gamma$ is smooth enough, it is well-known that one can reconstruct $\gamma$ from the DN map, see Sylvester and Uhlmann [57], Nachman [49, 50] and Novikov [52]. In dimension two, the DN map determines $\gamma \in L^{\infty}(\Omega)$, as shown by Astala and Päivärinta [4].

The above-mentioned theorems guarantee the unique determination of $\gamma$ from measurements on the whole surface of the body. However, it is often possible to measure only on part of the boundary. This is the case in medical and geophysical EIT since it is not practical to cover a patient or the Earth completely by electrodes. It is known in dimension three or higher, that if one knows the conductivity near the boundary one can reconstruct the conductivity from local voltage and current measurements [2]. This method seems to be difficult to implement numerically. In the general case, in dimension three or higher, it is shown in [41], that if one measures the voltage on an open subset of the boundary and measures the current flux in, roughly, the complement, one can determine uniquely the conductivity in the whole domain. There is no reconstruction procedure known at present for this result.

However, if we restrict ourselves to the anomaly detection problem, local measurements may be sufficient for obtaining crucial information about the conductivity. For example, cancerous breast tissue is known to differ significantly from healthy breast tissue in terms of conductivity [37]. Given local EIT data measured using electrodes placed on the breast, the problem is to find out whether there is a cancerous region (tumor) inside the breast, and if so, what is the approximate location of the tumor. See [59] and the references therein. Another relevant application of EIT is geophysical sensing for underground objects, see [36, 53, 54, 56].

We consider the problem of detection of inclusions in a two or three-dimensional body $\Omega$ from local boundary measurements. Let us assume that $\gamma(x)$ is a perturbation of known background conductivity $\gamma(x) \in C^{\infty}(\bar{\Omega})$. Namely, there exists an open subset $\Omega_{1} \subset \Omega$ such that $\bar{\Omega}_{1} \subset \Omega$ (this property is denoted by $\Omega_{1} \subset \subset \Omega$ ) and

$$
\gamma(x)= \begin{cases}\gamma_{1}(x), & x \in \Omega_{1}, \\ \gamma_{0}(x), & x \in \Omega_{0}:=\Omega \backslash \bar{\Omega}_{1},\end{cases}
$$


with $\gamma_{1}(x) \in L^{\infty}(\Omega)$ such that $\gamma(x) \geq c>0$ almost everywhere in $\Omega$. Let

$$
\Lambda_{0}:\left.f \rightarrow \gamma_{0}\left(\frac{\partial u}{\partial n}\right)\right|_{\partial \Omega}, \quad \Lambda:\left.f \rightarrow \gamma\left(\frac{\partial v}{\partial n}\right)\right|_{\partial \Omega}
$$

be the associated DN maps, where $v$ is the solution to (1.1) and $u$ solves equation (1.1) with $\gamma$ replaced by $\gamma_{0}$. We assume that $\gamma_{0}(x)$ is known on whole $\Omega$ and try to recover the location of $\Omega_{1}$ from the local knowledge of $\Lambda$. We prove: let a ball $B$ be given with center outside the convex hull of $\Omega$ and satisfying $(B \cap \partial \Omega) \subset \Gamma$. Then boundary measurements on $\Gamma$ with explicitly given Dirichlet data are enough to determine whether $\left(\bar{B} \cap \Omega_{1}\right)=\emptyset$ or not.

Let us briefly review existing theory on the inclusion detection problem. Unique determination from local boundary measurements has been studied by Isakov [31] and Ikehata $[21,25,26]$. Kohn and Vogelius show that piecewise analytic conductivities are uniquely determined by the DN map [42]. In some cases it is known that only one boundary measurement is sufficient to guarantee the uniqueness, see $[16,22,24,39,44]$ and the references therein. The reconstruction method presented in this paper is different from these previous studies.

There are two main motivations for our paper. The first one is the paper [33] where in dimension 3 approximate complex geometrical optics solutions concentrated near hemispheres are constructed for the conductivity equation. The article [33] uses hyperbolic geometry as we do in this paper. The second one is the work of Ikehata [27]. Using the inequality of Kang-Seo-Sheen [40] and Mittag-Leffler's function from the theory of functions of one complex variable, he gave a method to find the location of inclusions in the two-dimensional case. The associated numerical computation is given in [30]. The crucial feature of Mittag-Leffler's function is that it grows up exponentially in some sector and decays polynomially in the complement of the sector. In this paper we aim at generalizing these results in dimensions 2 and 3, making use of hemispheres instead of sectors. Note that since we probe with hemispheres, we are to some extent able to recover concave parts of inclusions.

Let us briefly describe our mathematical ideas. We embed the conductivity problem (1.1) into hyperbolic space. The role of straight lines in two dimensions (planes in three dimensions) in the Euclidean geometry is replaced by circles (spheres) in the hyperbolic geometry. Using this property we can construct solutions of equation (1.1) that are exponentially growing (with respect to a parameter) in a ball and exponentially decaying in its complement. This explains the term complex spherical waves in the title.

\subsection{Main theorems}

Throughout the paper we shall assume that

$\gamma_{1}(x)-\gamma_{0}(x)$ has a constant sign on $\Omega_{1}$, and for any $p \in \Omega_{1}$, there exist constants $C, \varepsilon>0$ such that

$$
C^{-1}<\left|\gamma_{1}(x)-\gamma_{0}(x)\right|<C \quad \text { if } \quad|x-p|<\varepsilon .
$$


Moreover $\partial \Omega_{1}$ is piecewise smooth.

Note that $\Omega_{1}$ is an open set and that by the assumption, we have either $\gamma(x)>\gamma_{0}(x)$ on $\Omega_{1}$ or $\gamma_{1}(x)<\gamma_{0}(x)$ on $\Omega_{1}$. Although our main purpose is to study discontinuous perturbations, we allow $\gamma(x)$ to be a continuous function. The inner product of $L^{2}(\partial \Omega)$ is denoted by $(,)_{\partial \Omega}$, the open ball with center $x_{0}$ and radius $R$ is denoted by $B\left(x_{0}, R\right)$, and $\operatorname{dis}(x, A)$ denotes the distance from the point $x$ to the set $A$.

Theorem 1.1. Take $x_{0}$ from the outside of the convex hull of $\Omega$. Let $\varepsilon>0$ be small enough so that $x_{0} \notin U_{\varepsilon}:=$ the $\varepsilon$-neighborhood of the convex hull of $\Omega$. Take an arbitrary constant $R>0$. Then there exists $u_{\tau}(x) \in C^{\infty}\left(U_{\varepsilon}\right)$ depending on a large parameter $\tau>0$ (and also on $R$ ) having the following properties.

(1) $u_{\tau}$ satisfies the equation

$$
\nabla \cdot\left(\gamma_{0}(x) \nabla u_{\tau}(x)\right)=0 \quad \text { on } \quad \Omega .
$$

(2) Let $K_{ \pm}$be any compact sets such that

$$
K_{+} \subset\left(U_{\mathcal{\varepsilon}} \cap B\left(x_{0}, R\right)\right), \quad K_{-} \subset\left(U_{\mathcal{\varepsilon}} \backslash \overline{B\left(x_{0}, R\right)}\right) .
$$

Then there exist constants $\delta>0$ and $T>0$ such that

$$
\int_{K_{+}}\left|u_{\tau}(x)\right|^{2} d x \geq e^{\delta \tau}, \quad \sup _{x \in K_{-}}\left|u_{\tau}(x)\right| \leq e^{-\delta \tau}, \quad \forall \tau>T .
$$

(3) Let $f_{\tau}(x)=\left.u_{\tau}\right|_{\partial \Omega}$. Then if $R<\operatorname{dis}\left(x_{0}, \partial \Omega_{1}\right)$, there exist $\delta>0$ and $T>0$ such that if $\gamma_{1}(x)>\gamma_{0}(x)$ on $\Omega_{1}$,

$$
0 \leq\left(\left(\Lambda-\Lambda_{0}\right) f_{\tau}, f_{\tau}\right)_{\partial \Omega}<e^{-\delta \tau}, \quad \forall \tau>T
$$

and if $\gamma_{1}(x)<\gamma_{0}(x)$ on $\Omega_{1}$,

$$
0 \leq\left(\left(\Lambda_{0}-\Lambda\right) f_{\tau}, f_{\tau}\right)_{\partial \Omega}<e^{-\delta \tau}, \quad \forall \tau>T .
$$

(4) If $R>\operatorname{dis}\left(x_{0}, \partial \Omega_{1}\right)$, there exist $\delta>0$ and $T>0$ such that if $\gamma_{1}(x)>\gamma_{0}(x)$ on $\Omega_{1}$

$$
\left(\left(\Lambda-\Lambda_{0}\right) f_{\tau}, f_{\tau}\right)_{\partial \Omega}>e^{\delta \tau}, \quad \forall \tau>T
$$

and if $\gamma_{1}(x)<\gamma_{0}(x)$ on $\Omega_{1}$

$$
\left(\left(\Lambda_{0}-\Lambda\right) f_{\tau}, f_{\tau}\right)_{\partial \Omega}>e^{\delta \tau}, \quad \forall \tau>T
$$

In order to deal with the case $R=\operatorname{dis}\left(x_{0}, \partial \Omega_{1}\right)$, we assume the following jump condition.

For any $p \in \partial \Omega_{1}$, there exists $\varepsilon>0$

such that $\left|\gamma_{1}(x)-\gamma_{0}(x)\right|>\varepsilon$ on $\Omega_{1} \cap B(p, \varepsilon)$.

Theorem 1.2. Let $\gamma_{0}$ and $\gamma_{1}$ satisfy (1.2). Take $R=\operatorname{dis}\left(x_{0}, \partial \Omega_{1}\right)$. Let $f_{\tau}$ be as in Theorem 1.1. Then if $\gamma_{1}(x)>\gamma_{0}(x)$ on $\Omega_{1}$,

$$
\liminf _{\tau \rightarrow \infty} \tau^{d-2}\left(\left(\Lambda-\Lambda_{0}\right) f_{\tau}, f_{\tau}\right)_{\partial \Omega}>0,
$$


and if $\gamma_{1}(x)<\gamma_{0}(x)$ on $\Omega_{1}$,

$$
\liminf _{\tau \rightarrow \infty} \tau^{d-2}\left(\left(\Lambda_{0}-\Lambda\right) f_{\tau}, f_{\tau}\right)_{\partial \Omega}>0 .
$$

It will be useful to give an approximate form of the above $f_{\tau}=\left.u_{\tau}\right|_{\partial \Omega}$. In the 3 -dimensional case, suppose that $\Omega \subset \subset \mathbb{R}_{+}^{3}=\left\{x=\left(x_{1}, x_{2}, x_{3}\right) ; x_{3}>0\right\}$ and $x_{0}=0$. Then if $\gamma_{0}(x)=1$, we have

$$
u_{\tau}(x) \simeq \sqrt{\frac{y_{3}}{x_{3}}} e^{-\tau y_{1}+i \tau y_{3}}
$$

where

$$
y_{1}=\frac{x_{1}^{2}+x_{2}^{2}+x_{3}^{2}-R^{2}}{\left(x_{1}+R\right)^{2}+x_{2}^{2}+x_{3}^{2}}, \quad y_{3}=\frac{2 x_{3} R}{\left(x_{1}+R\right)^{2}+x_{2}^{2}+x_{3}^{2}} .
$$

In the 2-dimensional case, suppose that $\Omega \subset \subset \mathbb{R}_{+}^{2}=\left\{x=\left(x_{1}, x_{2}\right) ; x_{2}>0\right\}, x_{0}=0$, and $\gamma_{0}(x)=1$. Then

$$
u_{\tau}(x) \simeq e^{-\tau y_{1}+i \tau y_{2}}
$$

where

$$
y_{1}=\frac{x_{1}^{2}+x_{2}^{2}-R^{2}}{\left(x_{1}+R\right)^{2}+x_{2}^{2}}, \quad y_{2}=\frac{2 x_{2} R}{\left(x_{1}+R\right)^{2}+x_{2}^{2}} .
$$

\subsection{Detection algorithm}

Suppose that $\gamma_{0} \equiv 1$. Then our detection algorithm in $\mathbb{R}^{d}$ with $d=2,3$ is as follows.

(i) Place the body $\Omega$ in the upper half space $\mathbb{R}_{+}^{d}$ so that $\partial \Omega$ has a positive distance from $\left\{x \in \mathbb{R}^{d} ; x_{d}=0\right\}$.

(ii) Draw a ball $B(0, R)$ such that $\overline{(B(0, R)} \cap \partial \Omega) \subset \Gamma$.

(iii) For large $\tau>0$, compute $I(\tau)=\left(\left(\Lambda-\Lambda_{0}\right) f_{\tau}, f_{\tau}\right)_{\partial \Omega}$.

(iv) If $I(\tau) \rightarrow 0$ as $\tau \rightarrow \infty$, we infer that $B(0, R)$ does not intersect the inclusion.

(v) If $I(\tau) \rightarrow \infty$ (or $-\infty$ ) as $\tau \rightarrow \infty$, we infer that $B(0, R)$ intersects the inclusion and $\gamma_{1}(x)>\gamma_{0}(x)$ (or $\left.\gamma_{1}(x)<\gamma_{0}(x)\right)$ on it.

In practical situations it is not possible to compute the limit $\tau \rightarrow \infty$ since measurement noise and the discrete nature of electrode data restrict $\tau$ to small values only. Inspired by the decay and growth estimates in claims (3) and (4) of Theorem 1.1 we suggest using only two finite values for $\tau$. We replace steps (iii), (iv) and (v) of the above algorithm by the following steps:

(iii) $^{\prime}$ Take $\tau_{1}<\tau_{2}$ and compute the corresponding inner products

$$
I_{1}:=\left(\left(\Lambda-\Lambda_{0}\right) f_{\tau_{1}}, f_{\tau_{1}}\right)_{\partial \Omega} \quad \text { and } \quad I_{2}:=\left(\left(\Lambda-\Lambda_{0}\right) f_{\tau_{2}}, f_{\tau_{2}}\right) \partial \Omega .
$$

Actually, we replace $f_{\tau}$ by its approximate form given in (1.3) or (1.4).

(iv) $^{\prime}$ If $I_{1} \geq I_{2}>0$ or $I_{1} \leq I_{2}<0$, we infer that $B(0, R)$ does not intersect the inclusion. 
(v) ${ }^{\prime}$ If $I_{2}>I_{1}>0$ (or $I_{2}<I_{1}<0$ ), we infer that $B(0, R)$ intersects the inclusion and $\gamma_{1}(x)>\gamma_{0}(x)\left(\right.$ or $\left.\gamma_{1}(x)<\gamma_{0}(x)\right)$ on it.

Of course, steps (iii) ${ }^{\prime}$, (iv) ${ }^{\prime}$ and (v)' give only approximate information whereas steps (iii), (iv) and (v) hold exactly. However, the necessity of using the modified steps is a consequence of the inherent ill-posedness of the inverse conductivity problem. Note that our algorithm does not involve the solution of any direct problem, so it is computationally effective.

In our numerical computation we use boundary data $g_{\tau}$ such that $g_{\tau}=f_{\tau}$ on $\Gamma$ and $g_{\tau}=0$ on $\partial \Omega \backslash \Gamma$. This choice leads to approximately correct results due to the exponential decay in $\tau$ of $f_{\tau}$ outside $\Gamma$. Theoretically it is desirable to prove the assertions (3) and (4) of Theorem 1.1 with $f_{\tau}$ replaced by $\chi_{\varepsilon}(x) f_{\tau}(x)$, where $\chi_{\varepsilon}(x) \in C_{0}^{\infty}\left(\mathbb{R}^{d}\right)$ satisfies $\chi_{\varepsilon}(x)=1$ on $B\left(x_{0}, R+\varepsilon / 2\right)$ and $\chi_{\varepsilon}(x)=0$ on $\mathbb{R}^{d} \backslash B\left(x_{0}, R+\varepsilon\right)$ with small $\varepsilon>0$. However for the moment we can prove it only when $\gamma \in C(\bar{\Omega})$ (see Theorem 2.7).

We can slightly improve our results. Even if we do not assume the constant sign of $\gamma_{1}(x)-\gamma_{0}(x)$ globally on $\Omega_{1}$, we can conclude the exponential decay of $\mid((\Lambda-$ $\left.\left.\Lambda_{0}\right) f_{\tau}, f_{\tau}\right) \mid$ when $R<\operatorname{dis}\left(x_{0}, \partial \Omega_{1}\right)$. To get a sharper information of dis $\left(x_{0}, \partial \Omega\right)$, the following local assumption on the sign is sufficient. Let $\mathscr{P}\left(x_{0}\right)$ be the set of points on $\partial \Omega_{1}$ where $B\left(x_{0}, R\right)$ first touches $\partial \Omega_{1}$. If there exists $\varepsilon>0$ such that $\gamma_{1}(x)-\gamma_{0}(x)$ has a constant sign on $\Omega_{1} \cap \mathscr{P}_{\varepsilon}\left(x_{0}\right)$, where $\mathscr{P}_{\varepsilon}\left(x_{0}\right)$ is the $\varepsilon$ neighborhood of $\mathscr{P}\left(x_{0}\right)$, our detection algorithm works in the following way. If $R$ is greater than but is very close to dis $\left(x_{0}, \partial \Omega_{1}\right)$, Theorem 1.1 (4) and Theorem 1.2 still hold. For instance, if $\gamma_{1}(x)-\gamma_{0}(x)$ has a constant sign on each connected component of $\Omega_{1}$, and if we know that $B\left(x_{0}, R\right)$ touches only one of these components, our algorithm works.

In dimension two, numerical detection of inclusions and other anomalies from measurements on the whole boundary has been discussed in $[3,6,9,10,11,13$, $15,17,18,19,28,29,30,34,38,43,44,45]$. While some of these algorithms may be modified to accept localized data, the present paper is the first to numerically demonstrate the recovery of two-dimensional electrical inclusions from localized measurements. We remark that a numerical algorithm for recovering the conductivity at the boundary from localized measurements is considered in [51]. In dimension three, reconstruction of features of conductivity from localized EIT measurements is studied in $[1,7,35,47,48,58]$. This paper presents a novel inclusion detection method for 3D EIT.

Our approach is independent of previous numerical works apart from the following connection. The harmonic function $\exp \left(i \tau\left(y_{2}+i y_{1}\right)\right)$ in (1.4) (introduced in the context of inverse conductivity problem already by Calderón in [12]) was used by Ikehata in [23] to prove that the convex hull of inclusion can be recovered from the DN map. Ikehata's method was implemented numerically in [29] and [10] simultaneously and independently. 


\subsection{Plan of paper}

We give the proofs of our theoretical results in section 2 and present a computational method based on the theory in section 3 . We test our detection algorithm in several two-dimensional situations using simulated noisy data in section 4 . We conclude our results in section 5. In appendix A we present elementary derivation of exact DN maps for a constant background and a layered medium; these results are used to validate our simulated data.

\section{Proof of main theorems}

\subsection{Reduction to the Schrödinger equation.}

Suppose $u$ satisfies the conductivity equation

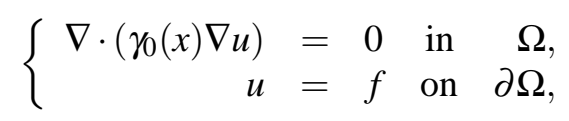

with the associated DN map

$$
\Lambda_{0} f=\left.\gamma_{0}(n \cdot \nabla) u\right|_{\partial \Omega}
$$

Then $u^{\prime}=\sqrt{\gamma_{0}} u$ satisfies

$$
\begin{cases}\left(-\Delta+q_{0}(x)\right) u^{\prime}=0 & \text { in } \quad \Omega, \quad q_{0}(x)=\frac{\Delta \sqrt{\gamma_{0}(x)}}{\sqrt{\gamma_{0}(x)}} \\ u^{\prime}=f^{\prime}:=\sqrt{\gamma_{0}} f & \text { on } \quad \partial \Omega\end{cases}
$$

with the associated DN map

$$
\Lambda_{01} f^{\prime}=\left.(n \cdot \nabla) u^{\prime}\right|_{\partial \Omega} .
$$

The DN maps are related as follows:

$$
\Lambda_{01}=\frac{1}{\sqrt{\gamma_{0}}} \Lambda_{0} \frac{1}{\sqrt{\gamma_{0}}}+\frac{(n \cdot \nabla) \sqrt{\gamma_{0}}}{\sqrt{\gamma_{0}}} .
$$

\subsection{Hyperbolic space}

Let $\mathbb{H}^{d}$ be the $d$-dimensional hyperbolic space realized in the upper-half space. Namely $\mathbb{H}^{d}=\mathbb{R}_{+}^{d}=\left\{\left(x, x_{d}\right) ; x \in \mathbb{R}^{d-1}, x_{d}>0\right\}$ equipped with the Riemannian metric

$$
d s^{2}=\left(x_{d}\right)^{-2}\left((d x)^{2}+\left(d x_{d}\right)^{2}\right) .
$$

The Laplace-Beltrami operator $\Delta_{g}$ on $\mathbb{H}^{d}$ is defined by

$$
\Delta_{g}=x_{d}^{2} \partial_{d}^{2}-(d-2) x_{d} \partial_{d}+x_{d}^{2} \Delta_{x},
$$

where $\partial_{d}=\partial / \partial x_{d}$ and $\Delta_{x}$ is the Euclidean Laplacian on $\mathbb{R}^{d-1}$. 


\subsection{Embedding to $\mathbb{H}^{3}$}

The main idea of the proof of Theorem 1.1 is to embed the Dirichlet problem (2.1) to the hyperbolic space $\mathbb{H}^{d}$. Let us begin with the 3-dimensional case. Without loss of generality, we assume that $x_{0}=0$ and that

$$
\Omega \subset \subset \mathbb{R}_{+}^{3}=\left\{\left(x, x_{3}\right) \in \mathbb{R}^{3} ; x \in \mathbb{R}^{2}, x_{3}>0\right\} .
$$

For a solution $u^{\prime}$ to the equation (2.1), we put $u^{\prime \prime}=\sqrt{x_{3}} u^{\prime}$. Then

$$
\begin{cases}\left(-\Delta_{g}+x_{3}^{2} q_{0}(x)-\frac{3}{4}\right) u^{\prime \prime}=0 & \text { in } \quad \Omega, \\ u^{\prime \prime}=f^{\prime \prime}:=\sqrt{x_{3} \gamma_{0}} f & \text { on } \quad \partial \Omega,\end{cases}
$$

where $\Delta_{g}=x_{3}^{2} \partial_{3}^{2}-x_{3} \partial_{3}+x_{3}^{2} \Delta_{x}$ is the Laplace-Beltrami operator on $\mathbb{H}^{3}$. The associated DN map is defined by

$$
\Lambda_{02} f^{\prime \prime}=\left.x_{3}(n \cdot \nabla) u^{\prime \prime}\right|_{\partial \Omega},
$$

where $n$ is the outer unit normal to $\partial \Omega$ with respect to the Euclidean metric $(d x)^{2}+$ $\left(d x_{3}\right)^{2}$ and $\nabla$ is the Euclidean gradient. Therefore

$$
\Lambda_{02}=x_{3} \sqrt{x_{3}} \Lambda_{01} \frac{1}{\sqrt{x_{3}}}+\sqrt{x_{3}}(n \cdot \nabla) \sqrt{x_{3}} .
$$

\subsection{Hyperbolic isometry on $\mathbb{H}^{3}$}

We are going to detect inclusions inside the sphere of radius $R$ centered at the origin, which is denoted by $S(0, R)$. We transform this sphere to a plane using hyperbolic isometry. We represent $\left(x_{1}, x_{2}, x_{3}\right) \in \mathbb{R}_{+}^{3}=\mathbb{H}^{3}$ by quaternions: $\mathbf{z}=$ $x_{1} \mathbf{1}+x_{2} \mathbf{i}+x_{3} \mathbf{j}$. Let us recall the representation of $\mathbf{z}$ by a $2 \times 2$ matrix:

$$
\mathbf{z}=x_{1} \mathbf{1}+x_{2} \mathbf{i}+x_{3} \mathbf{j}=\left(\begin{array}{cc}
x_{1}+i x_{3} & x_{2} \\
-x_{2} & x_{1}-i x_{3}
\end{array}\right) \text {. }
$$

It is well-known that for $\beta=\left(\begin{array}{ll}a & b \\ c & d\end{array}\right) \in S L(2, \mathbf{C})$, the transformation

$$
\mathbf{z} \rightarrow \beta \cdot \mathbf{z}=(a \mathbf{z}+b)(c \mathbf{z}+d)^{-1}
$$

is an isometry on $\mathbb{H}^{3}$. When $x_{3}=0$, this is a linear fractional transformation on $\mathbb{R}^{2} \times\{0\} \simeq \mathbf{C}$. We choose $\beta$ in such a way that this induced transformation maps the circle $S(0, R) \cap\left\{x_{3}=0\right\}$ to the line $x_{1}=x_{3}=0$, i.e.

$$
\beta=\left(\begin{array}{cc}
1 / \sqrt{2 R} & -\sqrt{R / 2} \\
1 / \sqrt{2 R} & \sqrt{R / 2}
\end{array}\right) .
$$

Then by the transformation (2.4), $S(0, R) \cap\left\{x_{3}>0\right\}$ is mapped to the semi-plane $\left\{x_{1}=0, x_{3}>0\right\}$. We show these facts by a direct computation. 
Lemma 2.1. The map $x \rightarrow y$ defined by

$$
\mathbf{w}=(\mathbf{z}-R \mathbf{1})(\mathbf{z}+R \mathbf{1})^{-1}=y_{1} \mathbf{1}+y_{2} \mathbf{i}+y_{3} \mathbf{j}
$$

is an isometry on $\mathbb{H}^{3}$, which maps

(1) the hemisphere $S(0, R) \cap\left\{x_{3}>0\right\}$ to the semi-plane $\left\{y_{1}=0, y_{3}>0\right\}$,

(2) the half-ball $B(0, R) \cap\left\{x_{3}>0\right\}$ to the quarter region $\left\{y_{1}<0, y_{3}>0\right\}$,

(3) the semi-plane $\left\{x_{2}=0, x_{3}>0\right\}$ to the semi-plane $\left\{y_{2}=0, y_{3}>0\right\}$.

Proof. The following maps are isometries on $\mathbb{H}^{d}$ :

- dilation: $\left(x, x_{d}\right) \rightarrow\left(\lambda x, \lambda x_{d}\right), \lambda>0$,

- translation: $\left(x, x_{d}\right) \rightarrow\left(x+x^{\prime}, x_{d}\right), x^{\prime} \in \mathbb{R}^{d-1}$,

- rotation: $\left(x, x_{d}\right) \rightarrow\left(R x, x_{d}\right), R \in O(d-1)$,

- inversion with respect to the sphere orthogonal to $\left\{x_{d}=0\right\}:\left(x, x_{d}\right) \rightarrow$ $\left(x / r^{2}, x_{d} / r^{2}\right), r^{2}=|x|^{2}+x_{d}^{2}$.

By (2.3) and (2.4) we have

$$
\left\{\begin{array}{l}
y_{1}=\frac{x_{1}^{2}+x_{2}^{2}+x_{3}^{2}-R^{2}}{\left(x_{1}+R\right)^{2}+x_{2}^{2}+x_{3}^{2}}, \\
y_{2}=\frac{2 x_{2} R}{\left(x_{1}+R\right)^{2}+x_{2}^{2}+x_{3}^{2}}, \\
y_{3}=\frac{2 x_{3} R}{\left(x_{1}+R\right)^{2}+x_{2}^{2}+x_{3}^{2}},
\end{array}\right.
$$

which then implies the lemma by a straightforward computation. Let us note that the inverse transform

$$
\mathbf{z}=R(\mathbf{w}+\mathbf{1})(-\mathbf{w}+1)^{-1}
$$

is given by

$$
\left\{\begin{array}{l}
x_{1}=R \frac{1-y_{1}^{2}-y_{2}^{2}-y_{3}^{2}}{\left(y_{1}-1\right)^{2}+y_{2}^{2}+y_{3}^{2}}, \\
x_{2}=R \frac{2 y_{2}}{\left(y_{1}-1\right)^{2}+y_{2}^{2}+y_{3}^{2}}, \\
x_{3}=R \frac{2 y_{3}}{\left(y_{1}-1\right)^{2}+y_{2}^{2}+y_{3}^{2}} .
\end{array}\right.
$$

\subsection{Embedding to $\mathbb{H}^{2}$}

The embedding of the 2-dimensional problem is much simpler than the 3dimensional case. Suppose $u$ satisfies in dimension two the equation

$$
(-\Delta+q) u^{\prime}=0 \quad \text { in } \Omega .
$$


Then multiplying by $x_{2}^{2}$, we get

$$
\left(-x_{2}^{2} \Delta+x_{2}^{2} q\right) u^{\prime}=0 \quad \text { in } \Omega .
$$

This is just the Schrödinger equation on $\mathbb{H}^{2}$.

\subsection{Hyperbolic isometry on $\mathbb{H}^{2}$}

In the 2-dimensional case, the above hyperbolic isometry is the usual linear fractional transformation.

Lemma 2.2. The map $x \rightarrow y$ defined by

$$
y_{1}+i y_{2}=\frac{\left(x_{1}+i x_{2}\right)-R}{\left(x_{1}+i x_{2}\right)+R}
$$

is an isometry on $\mathbb{H}^{2}$, which maps

(1) the hemi-circle $\left\{|x|=R, x_{2}>0\right\}$ to the half-line $\left\{y_{1}=0, y_{2}>0\right\}$,

(2) the half-disc $\left\{|x|<R, x_{2}>0\right\}$ to the quarter space $\left\{y_{1}<0, y_{2}>0\right\}$.

Proof. Recall that $y_{1}$ and $y_{2}$ are given by (1.5). The inverse transform is

$$
x_{1}=R \frac{1-y_{1}^{2}-y_{2}^{2}}{\left(y_{1}-1\right)^{2}+y_{2}^{2}}, \quad x_{2}=R \frac{2 y_{2}}{\left(y_{1}-1\right)^{2}+y_{2}^{2}} .
$$

Let $u^{*}$ and $\Lambda_{02}^{*}$ be $u$ and $\Lambda_{02}$ transformed by these isometries. Then the equations (2.2) and (2.6) are invariant. One should keep in mind that now one uses the new rectangular coordinates $x^{*}$ and the new Euclidean outer unit normal $n^{*}$ with respect to the new coordinate system. We use the same notation $\Omega, x, n$, respectively, for the transformed domain, the new variables and the new unit normal.

\subsection{Trial functions}

First let us recall the notion of complex geometrical optics solutions of the Schrödinger equation in $\mathbb{R}^{d}$.

For $s \in \mathbb{R}$, let $L^{2, s}$ be the weighted $L^{2}$-space defined by

$$
f \in L^{2, s} \Longleftrightarrow \int_{\mathbb{R}^{d}}(1+|x|)^{2 s}|f(x)|^{2} d x=\|f\|_{s}^{2}<\infty .
$$

For $\zeta \in \mathbf{C}^{d}$ such that $\zeta^{2}=\sum_{i=1}^{d} \zeta_{i}^{2}=0$, we define

$$
G_{0}(\zeta) f=(2 \pi)^{-d / 2} \int_{\mathbb{R}^{d}} \frac{e^{i x \cdot \xi}}{\xi^{2}+2 \zeta \cdot \xi} \widehat{f}(\xi) d \xi,
$$

$\widehat{f}(\xi)$ being the Fourier transform of $f$. Then for $s>1 / 2$, there exists a constant $C_{s}>0$ such that

$$
\left\|\partial_{x}^{\alpha} G_{0}(\zeta) f\right\|_{-s} \leq C_{s}|\zeta|^{|\alpha|-1}\|f\|_{s}, \quad|\zeta|>C_{s}, \quad|\alpha| \leq 1 .
$$


See $[49,57]$. For a bounded function of compact support $q(x)$, we define

$$
G_{q}(\zeta)=\left(1+G_{0}(\zeta) q\right)^{-1} G_{0}(\zeta) .
$$

Then $G_{q}(\zeta)$ also satisfies (2.7). We put

$$
w(\zeta)=e^{i x \cdot \zeta}-e^{i x \cdot \zeta} G_{q}(\zeta) q \cdot 1 .
$$

It has the following property.

Lemma 2.3. Let $w$ be as in (2.8). It satisfies:

(1) $(-\Delta+q) w(\zeta)=0$ on $\mathbb{R}^{d}$.

(2) For any compact set $D$ in $\mathbb{R}^{d}$, we have

$$
\left\|\partial_{x}^{\alpha}\left(e^{-i x \cdot \zeta} w(\zeta)\right)\right\|_{L^{2}(D)} \leq C|\zeta|^{|\alpha|-1} \quad \text { if } \quad|\alpha| \leq 1 .
$$

Now we construct our trial function in the hyperbolic space. Let us denote

$$
\begin{aligned}
& y=x_{3}, \quad x=\left(x_{1}, x_{2}\right) \quad \text { for } \quad d=3, \\
& y=x_{2}, \quad x=x_{1} \quad \text { for } \quad d=2 .
\end{aligned}
$$

We write $\zeta=(i \tau, 0, \cdots, 0, \tau)$ and define

$$
U(\tau)=x_{d}^{(d-2) / 2} w(\zeta) .
$$

Lemma 2.4. The function $U(\tau)$ defined above has the following properties.

(1) $\left(-y^{2} \Delta_{x, y}+(d-2) y \partial_{y}+V_{0}(x, y)-E_{d}\right) U(\tau)=0$ on $\Omega$, where $V_{0}(x, y)=y^{2} q(x, y)$ and $E_{d}=d(d-2) / 4$.

(2) Let $K_{ \pm}$be any compact sets such that

$$
K_{-} \subset \mathbb{R}_{+}^{d} \cup\left\{x_{1}>0\right\}, \quad K_{+} \subset \mathbb{R}_{+}^{d} \cup\left\{x_{1}<0\right\} .
$$

Then we have for some $\delta>0$

$$
\sum_{|\beta| \leq 1}\left|\partial_{x, y}^{\beta} U(\tau)\right| \leq e^{-\delta \tau} \quad \text { on } \quad K_{-}
$$

$$
\sum_{|\beta| \leq 1} \int_{K_{+}}\left|\partial_{x, y}^{\beta} U(\tau)\right|^{2} d x d y \geq e^{\delta \tau} .
$$

Proof. The assertion (1) follows from Lemma 2.3. By our construction

$$
U(\tau)=y^{(d-2) / 2} e^{-\tau x_{1}+i \tau y}(1+g(\tau)),
$$

where $g(\tau)$ satisfies

$$
\left\|\partial_{x, y}^{\beta} g(\tau)\right\|_{L^{2}(K)}=O\left(\tau^{|\beta|-1}\right)
$$

on any compact set $K \subset \mathbb{R}_{+}^{d}$. These facts prove (2.9) and (2.10).

As will be explained in the proof of Theorem 1.1, the asymptotic form of $u_{\tau}(x)$ in (1.3) follows from (2.11). If one needs a better expansion, one should take into account the Green operator $G_{0}(\zeta)$. Alternatively, one can use the Green operator in the hyperbolic space, as was done in [32] or [33]. 


\subsection{Proof of Theorem 1.1}

We use the notation

$$
(\psi, v)_{\Omega}=\int_{\Omega} \psi \bar{v} d x d y, \quad(f, g)_{\partial \Omega}=\int_{\partial \Omega} f \bar{g} d S,
$$

where $d S$ is the measure on $\partial \Omega$ induced from the Euclidean metric. First we recall the meaning of the solution of the equation (1.1). Let

$$
[\psi]_{\partial \Omega_{1}}=\operatorname{tr}_{+} \psi-\operatorname{tr}_{-} \psi
$$

be the jump of the function $\psi$ across $\partial \Omega_{1}$, where $\operatorname{tr}_{+}$and $\operatorname{tr}_{-}$denote the trace of $\psi$ on $\partial \Omega_{1}$ from the side of $\Omega_{1}$ and $\Omega_{0}$, respectively. For $f \in H^{3 / 2}(\partial \Omega)$, we put

$$
\mathscr{A}_{f}=\left\{\psi \in H^{2}\left(\Omega_{0}\right) \oplus H^{2}\left(\Omega_{1}\right) \mid \psi=f \text { on } \partial \Omega,[\psi]_{\partial \Omega_{1}}=0,\left[\gamma \frac{\partial \psi}{\partial n}\right]_{\partial \Omega_{1}}=0\right\} .
$$

By the solution of (1.1), we mean $v \in \mathscr{A}_{f}$ satisfying the equation $\nabla \cdot(\gamma(x) \nabla v(x))=$ 0 in $\Omega_{0} \cup \Omega_{1}$. The existence of $v$ follows from standard variational arguments and the uniqueness is an immediate consequence of the formula $(\gamma \partial v / \partial n, v)_{\partial \Omega}=$ $(\gamma \nabla v, \nabla v)_{\Omega}$. This formula in particular implies the following lemma.

\section{Lemma 2.5.}

$$
(\Lambda f, f)_{\partial \Omega}=\inf _{\psi \in \mathscr{A}_{f}}(\gamma \nabla \psi, \nabla \psi)_{\Omega}
$$

Our starting points are the following inequalities given in [40] (see also [20]).

Lemma 2.6. Let $f \in H^{3 / 2}(\partial \Omega)$, and $u$ be a solution to the boundary value problem

$$
\left\{\begin{array}{cc}
\nabla \cdot\left(\gamma_{0}(x) \nabla u\right)=0 & \text { in } \Omega, \\
u=f & \text { on } \partial \Omega .
\end{array}\right.
$$

Then for any positive $\gamma(x)$ and $\gamma_{0}(x)$, we have

$$
\begin{aligned}
\left(\left(\Lambda-\Lambda_{0}\right) f, f\right)_{\partial \Omega} & \leq\left(\left(\gamma-\gamma_{0}\right) \nabla u, \nabla u\right)_{\Omega}, \\
\left(\frac{\gamma_{0}}{\gamma}\left(\gamma-\gamma_{0}\right) \nabla u, \nabla u\right)_{\Omega} & \leq\left(\left(\Lambda-\Lambda_{0}\right) f, f\right)_{\partial \Omega} .
\end{aligned}
$$

Proof. Let $v$ be a solution to (1.1). By Green's formula, we have for $\varphi=v$ and $u$

$$
\begin{aligned}
& \left(\gamma \frac{\partial v}{\partial n}, \varphi\right)_{\partial \Omega}=(\gamma \nabla v, \nabla \varphi)_{\Omega} \\
& \left(\gamma_{0} \frac{\partial u}{\partial n}, \varphi\right)_{\partial \Omega}=\left(\gamma_{0} \nabla u, \nabla \varphi\right)_{\Omega} .
\end{aligned}
$$

Since $v=u=f$ on $\partial \Omega$, we then have by (2.14) and (2.15)

$$
\begin{aligned}
(\gamma \nabla v, \nabla v)_{\Omega} & =(\gamma \nabla v, \nabla u)_{\Omega}, \\
\left(\gamma_{0} \nabla u, \nabla v\right)_{\Omega} & =\left(\gamma_{0} \nabla u, \nabla u\right)_{\Omega} .
\end{aligned}
$$


Let us derive the following two formulas :

$$
\begin{aligned}
& (\gamma \nabla(v-u), \nabla(v-u))_{\Omega}+\left(\left(\gamma_{0}-\gamma\right) \nabla u, \nabla u\right)_{\Omega}=\left(\left(\Lambda_{0}-\Lambda\right) f, f\right)_{\partial \Omega} \\
& \left(\gamma_{0} \nabla(v-u), \nabla(v-u)\right)_{\Omega}+\left(\left(\gamma-\gamma_{0}\right) \nabla v, \nabla v\right)_{\Omega}=\left(\left(\Lambda-\Lambda_{0}\right) f, f\right)_{\partial \Omega}
\end{aligned}
$$

In fact, using (2.16) we have

$$
\begin{aligned}
& (\gamma \nabla(v-u), \nabla(v-u))_{\Omega} \\
= & -(\gamma \nabla u, \nabla v)_{\Omega}+\left(\gamma_{0} \nabla u, \nabla u\right)_{\Omega}+\left(\left(\gamma-\gamma_{0}\right) \nabla u, \nabla u\right)_{\Omega} \\
= & -(\Lambda f, f)_{\partial \Omega}+\left(\Lambda_{0} f, f\right)_{\partial \Omega}+\left(\left(\gamma-\gamma_{0}\right) \nabla u, \nabla u\right)_{\Omega},
\end{aligned}
$$

where in the second line we have used (2.14) and (2.15) with $\varphi=u$. This proves (2.17). The equality (2.18) is proven similarly.

Obviously, (2.17) implies (2.12). By completing the square we have

$$
\begin{aligned}
& \gamma_{0}|\nabla(v-u)|^{2}+\left(\gamma-\gamma_{0}\right)|\nabla v|^{2} \\
= & \gamma\left|\nabla v-\frac{\gamma_{0}}{\gamma} \nabla u\right|^{2}+\frac{\gamma_{0}}{\gamma}\left(\gamma-\gamma_{0}\right)|\nabla u|^{2} \\
\geq & \frac{\gamma_{0}}{\gamma}\left(\gamma-\gamma_{0}\right)|\nabla u|^{2},
\end{aligned}
$$

which together with (2.18) implies (2.13).

Let us note that Lemma 2.6 holds regardless of the sign of $\gamma(x)-\gamma(x)$.

Proof of Theorem 1.1. We prove the theorem in the case that $\chi_{1}(x)>\gamma_{0}(x)$ on $\Omega_{1}$. The other case is proved in a similar way. Let $d=3$. We take $x_{0}$ from the outside of the convex hull of $\Omega$. Without loss of generality, we assume that $x_{0}=0, \Omega \subset \subset \mathbb{R}_{+}^{3}$. Let $S(0, R)$ be the sphere of radius $R$ with center at the origin. By using Lemma 2.1, we map the hemisphere $S(0, R) \cap\left\{x_{3}>0\right\}$ to the semi-plane $\left\{y_{1}=0, y_{3}>0\right\}$ so that the half-ball $\left\{|x|<R, x_{3}>0\right\}$ is mapped to the region $\left\{y_{1}<0, y_{3}>0\right\}$. Now we follow the procedures given in sections 2.1, 2.3, 2.4 and 2.7:

$$
u \rightarrow u^{\prime}=\sqrt{\gamma_{0}} u \rightarrow u^{\prime \prime}=\sqrt{x_{3}} u^{\prime} \rightarrow\left(u^{\prime \prime}\right)^{*}(y)=u^{\prime \prime}(x) .
$$

This final $\left(u^{\prime \prime}\right)^{*}(y)$ satisfies the equation

$$
\left\{\begin{array}{c}
\left(-\Delta_{g}+V(y)\right)\left(u^{\prime \prime}\right)^{*}=0 \\
V(y)=x_{3}^{2} q_{0}(x)-\frac{3}{4}
\end{array}\right.
$$

We construct the solution $U(\tau)$ of this equation by Lemma 2.4. Transforming back, we put

$$
u_{\tau}(x)=\left(\gamma_{0}(x) x_{3}\right)^{-1 / 2} U\left(\tau, y_{1}, y_{2}, y_{3}\right)
$$

where $y_{1}, y_{2}, y_{3}$ are given by (2.5). Then $u_{\tau}$ solves the equation $\nabla \cdot\left(\gamma_{0}(x) \nabla u_{\tau}\right)=0$.

Note that $u_{\tau}$ is exponentially increasing in $\Omega \cap\{|x|<R\}$ and exponentially decreasing in $\Omega \cap\{|x|>R\}$. Let $f_{\tau}=\left.u_{\tau}\right|_{\partial \Omega}$. Then by Lemma 2.6, the behavior of $\left(\left(\Lambda-\Lambda_{0}\right) f_{\tau}, f_{\tau}\right)$ is controlled by $\left\|\nabla u_{\tau}\right\|_{L^{2}\left(\Omega_{1}\right)}$, which is exponentially decreasing or increasing accordingly as $\{|x|<R\} \cap \Omega_{1}=\emptyset$, or $\{|x|<R\} \cap \Omega_{1} \neq \emptyset$. This 
proves Theorem 1.1 for $d=3$. The two-dimensional case is proved in a similar manner.

\subsection{Proof of Theorem 1.2}

Proof of Theorem 1.2. As above we prove the case that $\gamma_{1}(x)>\gamma_{0}(x)$ on $\Omega_{1}$. We first consider the 3-dimensional case. Assume that the sphere $S(0, R)$ touches $\partial \Omega_{1}$ at $p$. By the use of hyperbolic isometry as in the proof of Theorem 1.1, one can assume that $p=\left(0,0, p_{3}\right)$ and $\Omega_{1} \subset\left\{x_{1}>0\right\}$ so that $u_{\tau}(x)$ behaves like $a(x) e^{-\tau x_{1}+i \tau x_{3}}$ near $p$, where $a(x) \in C^{\infty}$. Let us note that the condition (1.2) is invariant by hyperbolic isometries. One can then take an open cone $C_{p}$ with small positive opening and vertex $p$ such that $C_{p} \subset \Omega_{1}$. The area of the section $C_{p} \cap\left\{x_{1}=t\right\}$ is $C t^{2}$ with a constant $C>0$. We then have for some constant $\delta>0$

$$
\left\|\nabla u_{\tau}\right\|_{L^{2}\left(C_{p}\right)}^{2} \geq C \tau^{2} \int_{0}^{\delta} e^{-2 \tau x_{1}} x_{1}^{2} d x_{1}=\frac{C}{\tau} \int_{0}^{\tau \delta} e^{-2 y} y^{2} d y .
$$

By using (2.13) and (1.2), we then have

$$
\left(\left(\Lambda-\Lambda_{0}\right) f_{\tau}, f_{\tau}\right) \geq C\left\|\nabla u_{\tau}\right\|_{L^{2}\left(C_{p}\right)}^{2} \geq \frac{C}{\tau} \int_{0}^{\tau \delta} e^{-2 y} y^{2} d y .
$$

Theorem 1.2 then follows immediately. The 2-dimensional case is proved similarly.

\subsection{Localized measurements}

We end this section by the following theorem.

Theorem 2.7. Suppose $\gamma(x) \in C(\bar{\Omega})$. For a sufficiently small $\varepsilon>0$, let $\chi_{\varepsilon}(x) \in$ $C_{0}^{\infty}\left(\mathbf{R}^{d}\right)$ be such that $\chi_{\varepsilon}(x)=1$ on $B\left(x_{0}, R+\varepsilon / 2\right)$, and $\chi_{\varepsilon}(x)=0$ on $\left.\mathbf{R}^{d} \backslash B\left(x_{0}, R+\varepsilon\right)\right)$. We define $f_{\tau}^{(\infty)}(x)=\chi_{\varepsilon}(x) f_{\tau}(x)$. Then Theorem 1.1 (3) and (4) as well as Theorem 1.2 hold with $f_{\tau}$ replaced by $f_{\tau}^{(\infty)}$.

Proof. We prove the case that $\gamma_{1}(x)>\gamma_{0}(x)$ on $\Omega_{1}$. Since $\gamma(x) \in C(\bar{\Omega})$, Lemma 2.5 holds with $\mathscr{A}_{f}$ replaced by

$$
\mathscr{A}_{f}^{\prime}=\left\{\psi \in H^{1}(\Omega) ; \psi=f \text { on } \partial \Omega\right\} .
$$

Note that $\mathscr{A}_{f}^{\prime}$ does not depend on $\gamma$. Since $\gamma_{0} \leq \gamma$, Lemma 2.5 implies $\left(\Lambda_{0} f, f\right) \leq$ $(\Lambda f, f)$. Let $T=\Lambda-\Lambda_{0}$ and define $f_{\tau}^{(0)}=f_{\tau}-f_{\tau}^{(\infty)}$. Expanding

$$
\left(T\left(\varepsilon f_{\tau}^{(\infty)} \pm \varepsilon^{-1} f_{\tau}^{(0)}\right), \varepsilon f_{\tau}^{(\infty)} \pm \varepsilon^{-1} f_{\tau}^{(0)}\right) \geq 0,
$$

we have

$$
\left|\left(T f_{\tau}^{(\infty)}, f_{\tau}^{(0)}\right)+\left(T f_{\tau}^{(0)}, f_{\tau}^{(\infty)}\right)\right| \leq \varepsilon^{2}\left(T f_{\tau}^{(\infty)}, f_{\tau}^{(\infty)}\right)+\varepsilon^{-2}\left(T f_{\tau}^{(0)}, f_{\tau}^{(0)}\right) .
$$

Adding two (in)equalities

$$
\left(T f_{\tau}^{(\infty)}, f_{\tau}^{(\infty)}\right)+\left(T f_{\tau}^{(0)}, f_{\tau}^{(0)}\right)+\left(T f_{\tau}^{(\infty)}, f_{\tau}^{(0)}\right)+\left(T f_{\tau}^{(0)}, f_{\tau}^{(\infty)}\right)=\left(T f_{\tau}, f_{\tau}\right)
$$




$$
-\left(T f_{\tau}^{(\infty)}, f_{\tau}^{(0)}\right)-\left(T f_{\tau}^{(0)}, f_{\tau}^{(\infty)}\right) \leq \varepsilon^{2}\left(T f_{\tau}^{(\infty)}, f_{\tau}^{(\infty)}\right)+\varepsilon^{-2}\left(T f_{\tau}^{(0)}, f_{\tau}^{(0)}\right)
$$

we then have

$$
\left(1-\varepsilon^{2}\right)\left(T f_{\tau}^{(\infty)}, f_{\tau}^{(\infty)}\right) \leq\left(T f_{\tau}, f_{\tau}\right)+\left(\varepsilon^{-2}-1\right)\left(T f_{\tau}^{(0)}, f_{\tau}^{(0)}\right) .
$$

Adding

$$
\begin{aligned}
& \left(T f_{\tau}^{(\infty)}, f_{\tau}^{(\infty)}\right)+\left(T f_{\tau}^{(0)}, f_{\tau}^{(0)}\right)+\left(T f_{\tau}^{(\infty)}, f_{\tau}^{(0)}\right)+\left(T f_{\tau}^{(0)}, f_{\tau}^{(\infty)}\right)=\left(T f_{\tau}, f_{\tau}\right), \\
& -\left(T f_{\tau}^{(\infty)}, f_{\tau}^{(0)}\right)-\left(T f_{\tau}^{(0)}, f_{\tau}^{(\infty)}\right) \geq-\varepsilon^{2}\left(T f_{\tau}^{(\infty)}, f_{\tau}^{(\infty)}\right)-\varepsilon^{-2}\left(T f_{\tau}^{(0)}, f_{\tau}^{(0)}\right)
\end{aligned}
$$

we also have

$$
\left(1+\varepsilon^{2}\right)\left(T f_{\tau}^{(\infty)}, f_{\tau}^{(\infty)}\right)+\left(1+\varepsilon^{-2}\right)\left(T f_{\tau}^{(0)}, f_{\tau}^{(0)}\right) \geq\left(T f_{\tau}, f_{\tau}\right) .
$$

Let $u_{\tau}^{(0)}=\left(1-\chi_{\varepsilon}(x)\right) u_{\tau}$. Then $u_{\tau}^{(0)} \in \mathscr{A}_{f_{\tau}^{(0)}}^{\prime}$, hence by Lemma 2.5

$$
\left(\Lambda f_{\tau}^{(0)}, f_{\tau}^{(0)}\right)_{\partial \Omega} \leq\left(\gamma \nabla u_{\tau}^{(0)}, \nabla u_{\tau}^{(0)}\right)_{\Omega} .
$$

By Theorem 1.1 (2), the right-hand side is exponentially decreasing in $\tau$. Replacing $\gamma$ by $\gamma_{0}$, we also have that $\left(\Lambda_{0} f_{\tau}^{(0)}, f_{\tau}^{(0)}\right)$ is exponentially decreasing. Therefore for some $\delta>0$,

$$
\left|\left(T f_{\tau}^{(0)}, f_{\tau}^{(0)}\right)\right| \leq C e^{-\delta \tau} .
$$

Suppose $R<\operatorname{dis}\left(x_{0}, \partial \Omega\right)$. Then in view of Theorem 1.1 (3), (2.19) and (2.21), we see that $\left(T f_{\tau}^{(\infty)}, f_{\tau}^{(\infty)}\right)$ is exponentially decreasing. If $R>\operatorname{dis}\left(x_{0}, \partial \Omega\right)$. Then in view of Theorem 1.1 (4), (2.20) and (2.21), we see that $\left(T f_{\tau}^{(\infty)}, f_{\tau}^{(\infty)}\right)$ is exponentially increasing. If $R=\operatorname{dis}\left(x_{0}, \partial \Omega\right)$, by virtue of Theorem 1.2, (2.20) and (2.21), Theorem 1.2 holds with $f_{\tau}$ replaced by $f_{\tau}^{(\infty)}$.

\section{Computational methods}

In this section we introduce a computational framework for practical testing of our theoretical results. The framework will be put into use in section 4 .

\subsection{Simulation of data}

Recalling that our domain $\Omega$ must be away from the horizontal line $\left\{x_{2}=0\right\}$, we take $\Omega$ to be the square

$$
\Omega=\left\{\left(x_{1}, x_{2}\right):-1 \leq x_{1} \leq 1, \quad \varepsilon \leq x_{2} \leq 2+\varepsilon\right\} \subset \mathbb{R}^{2},
$$

$\varepsilon>0$ being a small constant, and denote the bottom boundary of $\Omega$ by $\Gamma$ :

$$
\Gamma=\left\{\left(x_{1}, \varepsilon\right):-1<x_{1}<1\right\} .
$$



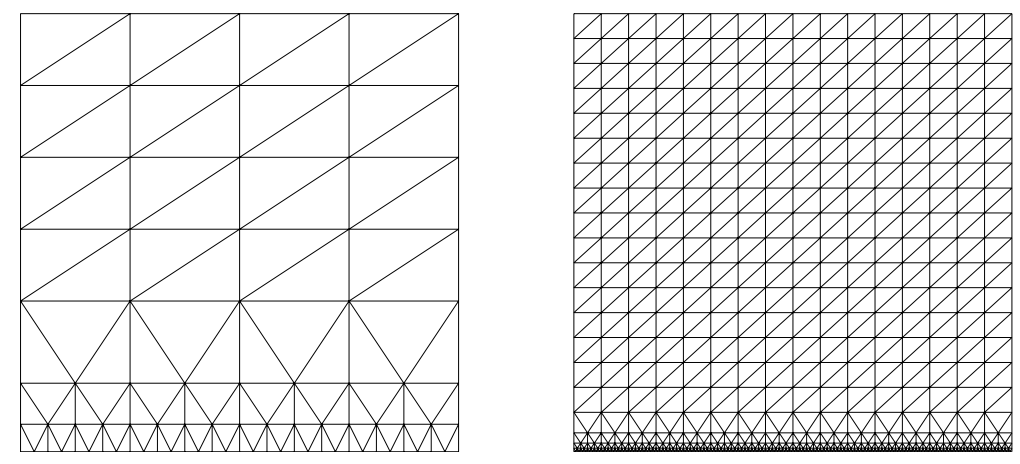

FIgURE 3.1. Two examples of our tailored FEM meshes. We refine the mesh near $\Gamma$ to allow numerical differentiation of the solution on $\Gamma$ to evaluate the DN map. The mesh has $2^{M}+1$ nodes on the top boundary and $2^{N}+1$ nodes on the bottom boundary. Left: the mesh with $M=2$ and $N=5$. Right: $M=4$ and $N=8$. In our actual computations we use $M=6$ and $N=12$.

In all our numerical examples we assume that the background conductivity is constant: $\gamma_{0} \equiv 1$. We assume that we can measure only on $\Gamma$ and use the Dirichlet data

$$
f_{\tau} \text { on } \Gamma, \quad 0 \text { on } \partial \Omega \backslash \Gamma .
$$

Exponential decay of $f_{\tau}$ outside $\Gamma$ ensures that the error caused by the truncation (3.3) is small.

For the computation of DN maps we use two approaches. First, for homogeneous and layered media we can represent the DN map exactly, as explained in Appendix A. Second, for general conductivities we use the Finite Element Method (FEM). We solve a given Dirichlet problem using a FEM mesh of the type depicted in Figure 3.1, and use finite differences to approximate the normal derivative of the solution on $\Gamma$. We validate our FEM code by comparing the results from the two approaches for simple examples with layered media.

We add noise to the simulated data as follows. Define a random function $\eta$ : $[-1,1] \rightarrow \mathbb{C}$ by

$$
\eta(t)=\sum_{j=-32}^{32}\left(a_{j}+i b_{j}\right) e^{i j t \pi / 2},
$$

where $a_{j}, b_{j} \sim \mathscr{N}(0,1)$ are normally distributed random numbers. The number 32 in (3.4) is chosen to roughly model a collection of 32 electrodes on the bottom boundary of $\Omega$ (the function $\exp (i t 32 \pi / 2)$ oscillates with the maximum frequency 
that can be effectively approximated by 32 equidistant point values on $\Gamma$ ). Measurements noise is modelled by replacing $\Lambda f_{\tau}$ by $\Lambda f_{\tau}+c \eta$ with noise amplitude

$$
c:=\frac{A\left\|\Lambda f_{\tau}\right\|_{\infty}}{\|\eta\|_{\infty}}
$$

where $A>0$ is suitably chosen.

\subsection{Plan of detection}

We cannot probe the whole square $\Omega$ by applying our method to measurements done on the bottom $\Gamma$. Let us define the maximal subset $Q_{\max } \subset \Omega$ available for sensing. For any point $x \in \mathbb{R}^{2} \backslash \Omega$, define

$$
\left.R_{\max }(x):=\sup \{R \geq 0 \mid \overline{(B(x, R)} \cap \partial \Omega) \subset \Gamma\right\} .
$$

The number $R_{\max }(x)$ gives the radius of the maximal disc centered at $x$ ensuring that Dirichlet data supported in $B\left(x, R_{\max }(x)\right) \cap \partial \Omega$ is also supported in $\Gamma$. Set

$$
Q_{\max }:=\bigcup_{x \in \Gamma}\left(B\left(x, R_{\max }(x)\right) \cap \Omega\right) .
$$

Of course, in Theorem 1.1 the center of the sensing disc must be chosen from outside of $\bar{\Omega}$, and thus we cannot use the discs $B\left(x, R_{\max }(x)\right)$ with $x \in \Gamma$. However, we can choose a point $\tilde{x} \in \mathbb{R}^{2} \backslash \bar{\Omega}$ arbitrarily close to $x$ and use Theorem 1.1 to check whether the disc $B\left(\tilde{x}, R_{\max }(\tilde{x})\right)$ intersects the inclusion or not. The set $Q_{\max }$ is the limit case.

Further, given an inclusion $\Omega_{1} \subset \subset \Omega$, let us define the largest region $Q_{\text {best }}$ on which we can conclude the absence of inclusions by the measurements on $\Gamma$. Set

$$
\left.R_{\text {best }}(x):=\sup \{R \geq 0 \mid \overline{(B(x, R)} \cap \partial \Omega) \subset \Gamma,\left(\overline{B(x, R)} \cap \bar{\Omega}_{1}\right)=\emptyset\right\},
$$

and define

$$
Q_{\text {best }}:=\bigcup_{x \in \Gamma}\left(B\left(x, R_{\text {best }}(x)\right) \cap \Omega\right) .
$$

Practical measurements are noisy, however, and we cannot expect to recover the set $Q_{\text {best }}$ from measured data. In practice, reconstructions are computed using the following algorithm. Choose two parameters $\tau_{1}<\tau_{2}$ and a finite set of points $x^{(j)} \in \mathbb{R}^{2} \backslash \bar{\Omega}$ with $j=1,2,3, \ldots, J$. For each $j$ choose a finite set of radii $0<$ $R(j, k)<R_{\max }\left(x^{(j)}\right)$ for $k=1,2,3, \ldots, K$. Define inner products

$$
I_{1}(j, k):=\left(\left(\Lambda-\Lambda_{0}\right) f_{\tau_{1}}, f_{\tau_{1}}\right)_{\Gamma} \quad \text { and } \quad I_{2}(j, k):=\left(\left(\Lambda-\Lambda_{0}\right) f_{\tau_{2}}, f_{\tau_{2}}\right)_{\Gamma}
$$

where $f_{\tau}=\left.u_{\tau}\right|_{\partial \Omega}$ depends on $x^{(j)}$ and $R(j, k)$ through formulas (1.4) and (1.5). For each point $x^{(j)}$, define the largest disc not intersecting the inclusion by formula

$$
R_{\mathrm{rec}}(j):=\max _{1 \leq k \leq K}\left\{R(j, k) \mid I_{1}(j, k) \geq I_{2}(j, k)\right\} .
$$




\section{Geometry}

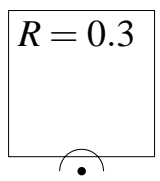

$R=0.5$
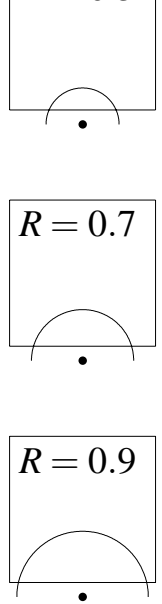

Dirichlet data
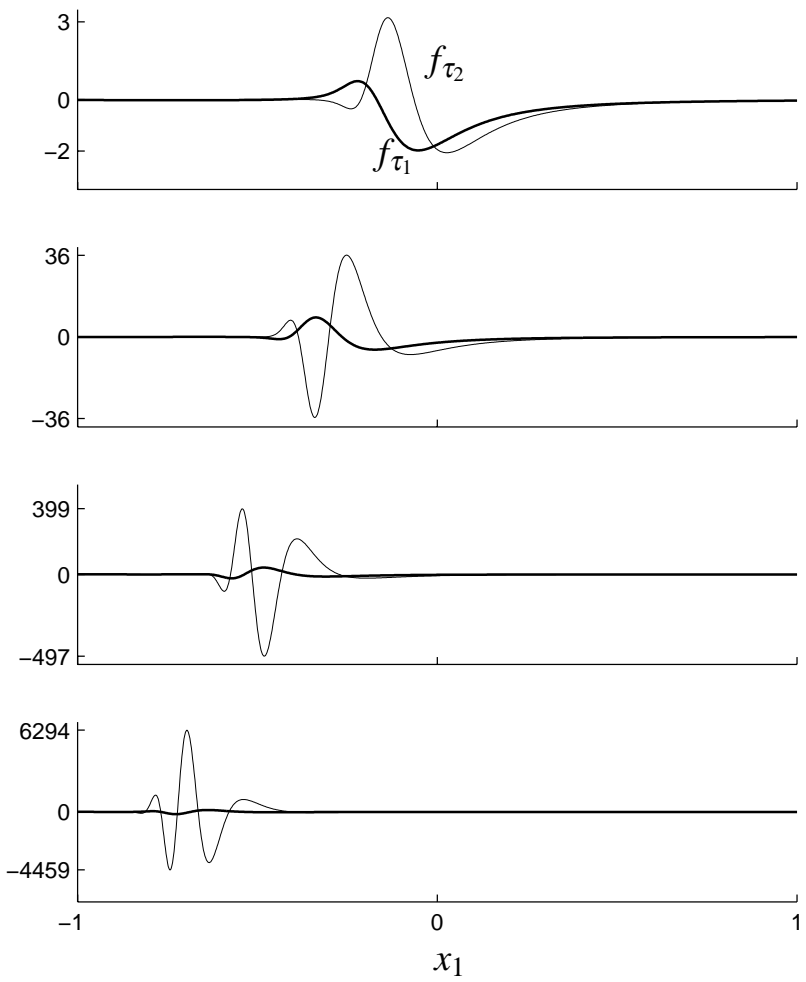

FIGURE 3.2. Examples of the Dirichlet data used in the detection algorithm with $\tau_{1}=1.5$ and $\tau_{2}=2.5$. Left column shows the choices of sensing discs. The plots in the right column show the corresponding Dirichlet data on $\Gamma$. Note the asymmetry of the functions with respect to the interval $B(x, R) \cap \Gamma$.

Then we can set

$$
D_{\mathrm{rec}}:=\bigcup_{1 \leq j \leq J}\left(B\left(x^{(j)}, R_{\mathrm{rec}}(j)\right) \cap \Omega\right),
$$

and conclude that $D_{\text {rec }}$ does not intersect the inclusion.

Remark that in Section 1.3 we describe the algorithm in the form that the center of the sensing disc is at the origin and the domain is translated in the upper half plane. Due to translation invariance the approach of this section is equivalent.

At this point we introduce an enhancement of the numerical reconstruction algorithm. Namely, as can be seen in Figure 3.2, the Dirichlet data $f_{\tau}$ defined using hyperbolic geometry is strongly asymmetric. So we expect the above algorithm to perform differently in practice according to the location of inclusions. (This expectation is confirmed by numerical experiments.) To overcome this problem we assume that the domain $\Omega$ is symmetric with respect to the $x_{2}$-axis: $\Omega=$ 
$\left\{\left(x_{1}, x_{2}\right) \mid\left(-x_{1}, x_{2}\right) \in \Omega\right\}$. We define the set $D_{\text {rec }}^{\prime}$ similarly to $D_{\text {rec }}$ above but corresponding to the reflected conductivity $\gamma\left(x_{1}, x_{2}\right):=\gamma\left(-x_{1}, x_{2}\right)$. Then we denote

$$
D_{\text {rec }}^{\prime \prime}:=\left\{\left(x_{1}, x_{2}\right) \mid\left(-x_{1}, x_{2}\right) \in D_{\text {rec }}^{\prime}\right\}
$$

and define the symmetrized reconstruction as

$$
Q_{\text {rec }}:=D_{\text {rec }} \cup D_{\text {rec }}^{\prime \prime} \text {. }
$$

Our final reconstruction result is that the set $Q_{\text {rec }}$ does not intersect the inclusion.

We use the following quantity for measuring relative error of reconstructions:

$$
\frac{\mu\left(Q_{\text {best }} \backslash Q_{\text {rec }}\right)+\mu\left(Q_{\text {rec }} \backslash Q_{\text {best }}\right)}{\mu\left(Q_{\text {best }}\right)} \cdot 100 \%,
$$

where $\mu$ stands for area (Lebesgue measure in $\mathbb{R}^{2}$ ).

\section{Numerical results}

\subsection{Layered medium}

Let $\sigma=4$ and $0<h<2$. Take $\Omega$ as in (3.1) with $\varepsilon=0.2$ and define a discontinuous conductivity $\gamma$ on $\Omega$ :

$$
\gamma\left(x_{1}, x_{2}\right)= \begin{cases}\sigma & \text { for } x_{2}>h+\varepsilon \\ 1 & \text { for } x_{2} \leq h+\varepsilon\end{cases}
$$

Here $\gamma$ models a material with two layers of different conductivities divided by an interface at $x_{2}=h+\varepsilon$.

In this simple situation the conductivity is completely characterized by one real number, namely the interface position $h$. In the case $h<R_{\max }(0)-\varepsilon$, it is enough to know $R_{\text {best }}(0)$. We study our detection algorithm by recovering $R_{\text {rec }}(0)$ from measured data corresponding to several choices of $h$ and computing the relative error

$$
\frac{\left|R_{\text {best }}(0)-R_{\text {rec }}(0)\right|}{R_{\text {best }}(0)} \cdot 100 \% .
$$

Figure 4.1(a) illustrates the geometry of our setup: for fixed interface $h$, we take several discs with center at the origin and varying radii.

We take $\tau_{1}=1.5$ and $\tau_{2}=2.5$ and compute the inner products (3.8) using trapezoidal rule. See Figure 4.2 for the results.

To ensure that our FEM-based solver is correct, we compare the numerical normal derivatives to the ones calculated using the exact eigenvalues of DN maps given in Appendix A. The relative $L^{\infty}([-1,1])$ difference between normal derivatives computed with the two methods is less than $0.1 \%$ for a representative set of layered conductivities and for sinusoidal Dirichlet data within relevant frequency range. We conclude that we can trust our FEM code.

Next we introduce noise in the data. We choose $A=0.0001$ in formula (3.5); this corresponds to $0.01 \%$ noise level. Reconstructions are shown in Figure 4.2. 

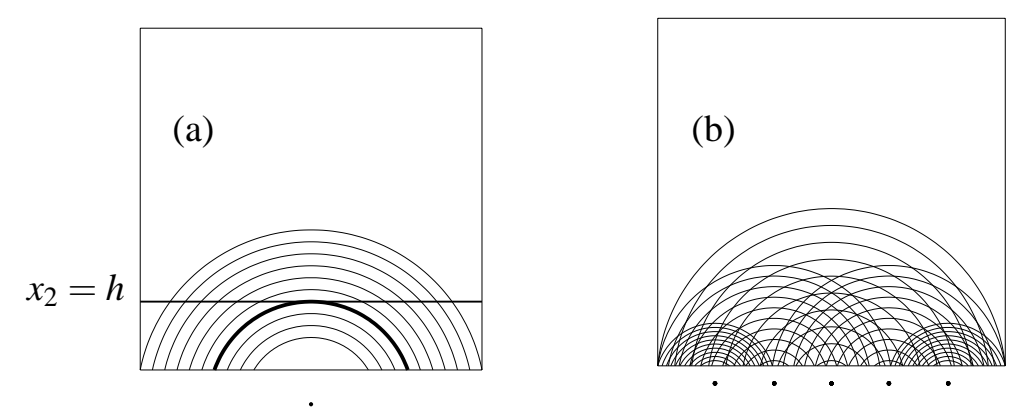

FIGURE 4.1. Collection of discs for probing the square domain. Black dots denote centers of discs. (a) Discs for probing layered media. The maximal disc $B\left(0, R_{\text {best }}(0)\right)$ that touches the upper layer is drawn with thick line. (b) Discs for probing inclusions.

\subsection{Inclusions in homogeneous background}

We choose test conductivities having inclusions in homogeneous background. We again take the background conductivity to equal 1, and the inclusions are chosen to be various geometrical shapes with conductivity 4 . The example conductivities are shown in the left column of Figure 4.3.

Figure 4.1(b) shows one possible collection of discs for probing the domain. We use somewhat more discs than shown in Figure 4.1(b). See Figure 4.3 for reconstructions from ideal and noisy data. The percentages are given by formula (3.12).

\section{Conclusion}

We present a novel algorithm for detecting inclusions in known background conductivity from localized boundary measurements. Our method allows (partial) recovery of concave features of inclusions since we use spheres to probe the unknown medium. We justify the algorithm theoretically using hyperbolic geometry. While the numerical examples in this paper are two-dimensional, the algorithm is applicable in three-dimensional situations as well.

Although the reconstruction Theorem 1.1 holds only asymptotically for large enough $\tau$, for practical reasons we must take $\tau<3$. Namely, using large values of $\tau$ would require the application of Dirichlet data with impractical amplitude and frequency of spatial oscillation. However, we achieve useful reconstructions that

(1) use Dirichlet data suitable for practical electrode measurements,

(2) are robust against measurement noise, and

(3) require very little computational effort (in particular, our method is not iterative and no direct problems need to be solved).

Our numerical studies suggest that inclusions relatively close to the boundary can be detected even from noisy data. However, we cannot probe reliably very deep 


\section{Geometry}
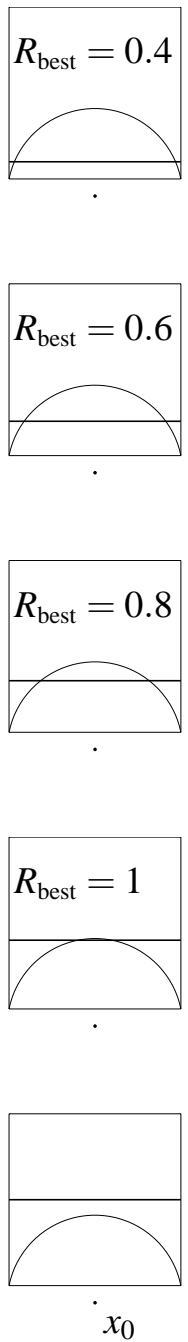

Reconstruction from ideal data
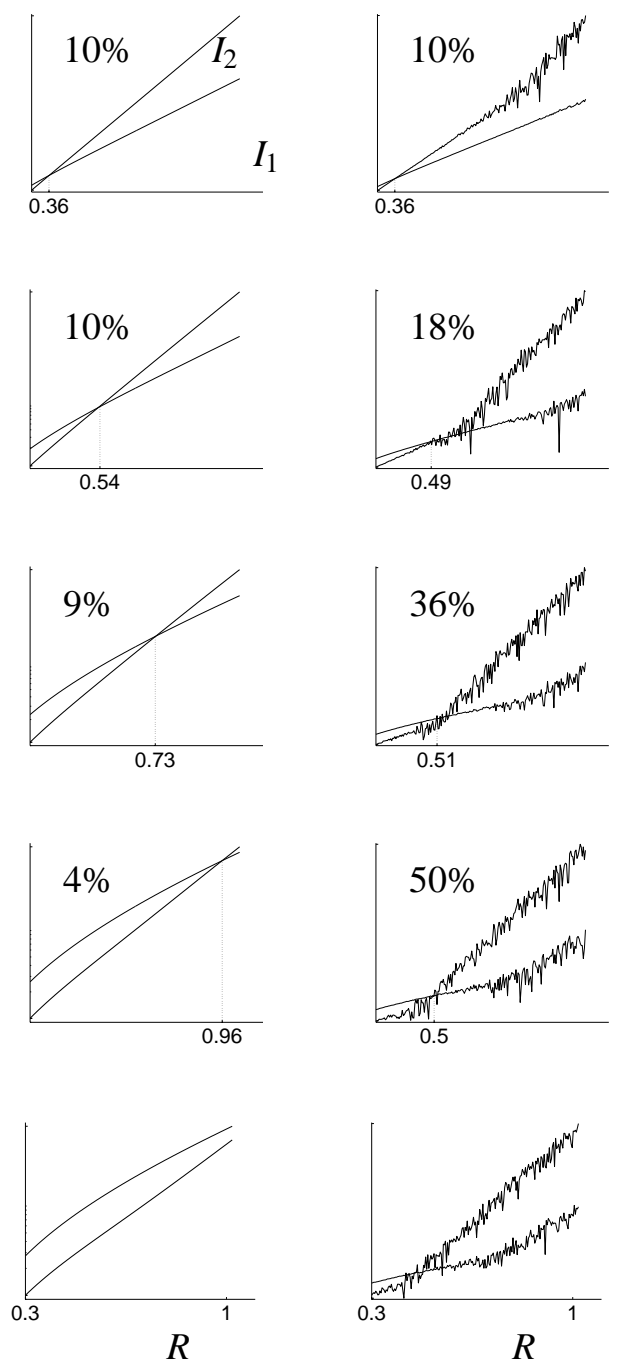

FIGURE 4.2. Left column: Geometry of layered medium examples. Thick horizontal line indicates the interface between two layers with different conductivity. $R_{\text {best }}=R_{\text {best }}(0)$ denotes the radius of the largest sensing disc that does not intersect the upper conductivity layer. Middle column: The logarithms of inner products $I_{1}$ and $I_{2}$ are plotted as functions of $R$. The percentage gives relative error (4.2). Right column: same plots than in middle column except that noise is added to the simulated measurements. 
Original

conductivity
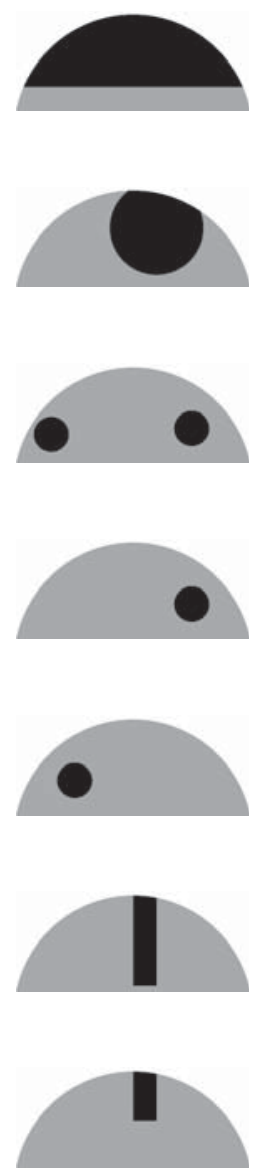

$Q_{\text {best }}$
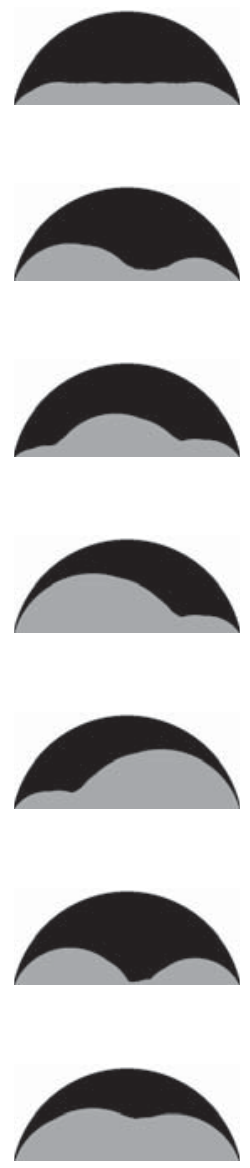

Reconstruction from ideal data
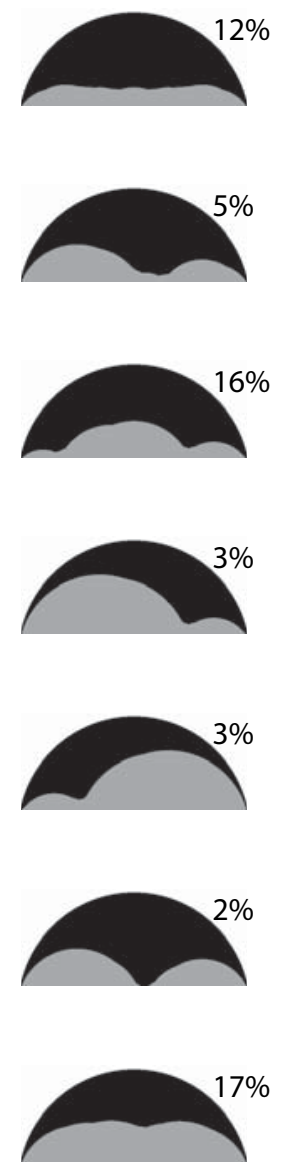

Reconstruction from data with $0.01 \%$ noise
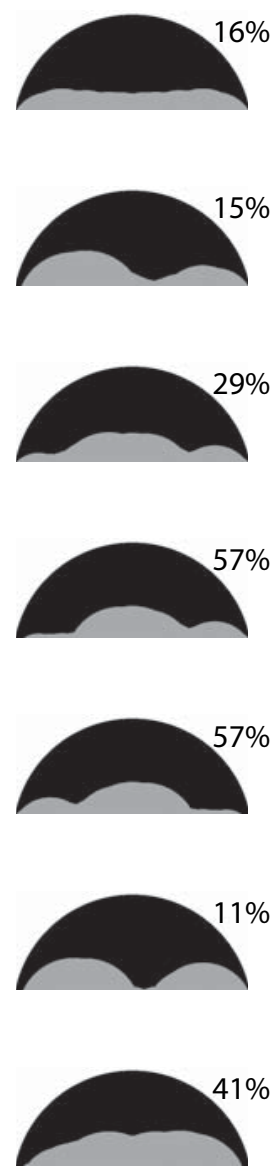

FIGURE 4.3. Each row of images demonstrates reconstructions of an example conductivity containing inclusions. All functions are plotted only inside the maximal set $Q$ max as defined in (3.6), rather than in the full square domain $\Omega$. First column: true conductivity. Gray is homogeneous background, black is inclusion. Second column: the largest region $Q$ best on which we can conclude the absence of inclusions by the measurements on $\Gamma$, see (3.7). Third column: the set $Q$ rec as defined in (3.11) reconstructed from simulated boundary data with no noise. The percentage is the relative error defined in (3.12). Fourth column: the same as third column but computed using noisy simulated boundary data. 
into the unknown medium. This is in accordance with the usual intuition about EIT: details in conductivity that are far away from the boundary are more difficult to recover than details near the boundary.

Our method finds applications in medical imaging, industrial process monitoring and nondestructive testing.

\section{Appendix: Exact DN map for layered medium in dimension two}

\section{A.1 DN map for homogeneous conductivity}

Let $\Omega$ and $\Gamma$ be given by (3.1) and (3.2), respectively, with $\varepsilon=0$. We want to solve the Dirichlet problem

$$
\left\{\begin{array}{lll}
\Delta u=0 & \text { in } \quad \Omega, \\
u=\varphi_{k} & \text { on } \Gamma, \\
u=0 & \text { on } & \partial \Omega \backslash \Gamma,
\end{array}\right.
$$

where $\varphi_{k}\left(x_{1}\right)=\sin \left(k \pi\left(x_{1}+1\right) / 2\right)$ for $-1 \leq x_{1} \leq 1$ and $k \in \mathbb{Z}$. We use the ansatz

$$
\text { (A.2) } \quad u\left(x_{1}, x_{2}\right)=\left[A \exp \left(\frac{k \pi}{2} x_{2}\right)+B \exp \left(-\frac{k \pi}{2} x_{2}\right)\right] \sin \left(\frac{k \pi}{2}\left(x_{1}+1\right)\right) \text {. }
$$

Now at the interval specified by $x_{2}=2$ and $-1 \leq x_{1} \leq 1$ the function $u$ must vanish, leading to $A \exp (k \pi)+B \exp (-k \pi)=0$. Further, $\left.u\right|_{\Gamma}=\varphi_{k}$ gives $A+B=1$. Thus

$$
A=\frac{1}{1-e^{2 k \pi}}, \quad B=-\frac{e^{2 k \pi}}{1-e^{2 k \pi}} .
$$

The functions $\varphi_{k}$ are eigenfunctions for $\Lambda_{0}$ :

$$
\Lambda_{0} \varphi_{k}=-\left.\frac{\partial u}{\partial x_{2}}\right|_{x_{2}=0}=-\frac{k \pi}{2}(B-A) \varphi_{k}=k \pi\left(\frac{1+e^{-2 k \pi}}{1-e^{-2 k \pi}}\right) \varphi_{k} .
$$

\section{A.2 DN map for layered medium}

We want to solve

$$
\left\{\begin{array}{lll}
\nabla \cdot(\gamma \nabla v)=0 & \text { in } & \Omega, \\
v=\varphi_{k} & \text { on } & \Gamma, \\
v=0 & \text { on } & \partial \Omega \backslash \Gamma,
\end{array}\right.
$$

where $\gamma$ is defined by (4.1) with $\varepsilon=0$. The solution $v$ is harmonic in the open sets $\Omega \cap\left\{x_{2}>h\right\}$ and $\Omega \cap\left\{x_{2}<h\right\}$, and both $v$ and $\gamma(\partial v / \partial n)$ are continuous at the interface.

We will solve (A.3) with the ansatz

$$
v\left(x_{1}, x_{2}\right)= \begin{cases}{\left[C \exp \left(\frac{k \pi}{2} x_{2}\right)+D \exp \left(-\frac{k \pi}{2} x_{2}\right)\right] \sin \left(\frac{k \pi}{2}\left(x_{1}+1\right)\right)} & \text { for } \quad x_{2} \geq h, \\ {\left[E \exp \left(\frac{k \pi}{2} x_{2}\right)+F \exp \left(-\frac{k \pi}{2} x_{2}\right)\right] \sin \left(\frac{k \pi}{2}\left(x_{1}+1\right)\right)} & \text { for } \quad x_{2}<h .\end{cases}
$$


At the interval specified by $x_{2}=2$ and $-1 \leq x_{1} \leq 1$ the function $v$ must vanish, giving $C \exp (k \pi)+D \exp (-k \pi)=0$. Continuity of $v$ at the interface yields

$$
C \exp \left(\frac{k \pi h}{2}\right)+D \exp \left(-\frac{k \pi h}{2}\right)=E \exp \left(\frac{k \pi h}{2}\right)+F \exp \left(-\frac{k \pi h}{2}\right),
$$

and continuity of $\gamma(\partial v / \partial n)$ at the interface gives

$$
\sigma C \exp \left(\frac{k \pi h}{2}\right)-\sigma D \exp \left(-\frac{k \pi h}{2}\right)=E \exp \left(\frac{k \pi h}{2}\right)-F \exp \left(-\frac{k \pi h}{2}\right) .
$$

The condition $\left.v\right|_{\Gamma}=\varphi_{k}$ leads to $F=1-E$. Finally, we get $D=-C \exp (2 k \pi)$ and

$$
\begin{aligned}
C & =\frac{e^{-k \pi h}}{\left(1+e^{-k \pi h}\right)\left(1-e^{k \pi(2-h)}\right)-\sigma\left(1-e^{-k \pi h}\right)\left(1+e^{k \pi(2-h)}\right)}, \\
E & =\frac{e^{-k \pi h}\left\{\sigma\left(1+e^{k \pi(2-h)}\right)-\left(1-e^{k \pi(2-h)}\right)\right\}}{2\left\{\sigma\left(1-e^{-k \pi h}\right)\left(1+e^{k \pi(2-h)}\right)+\left(1+e^{-k \pi h}\right)\left(1-e^{k \pi(2-h)}\right)\right\}} .
\end{aligned}
$$

The functions $\varphi_{k}$ are eigenfunctions for $\Lambda$ :

$$
\Lambda \varphi_{k}=-\left.\frac{\partial v}{\partial x_{2}}\right|_{x_{2}=0}=-\frac{k \pi}{2}(E-F) \varphi_{k}=\frac{k \pi}{2}\left(\frac{1}{2}-2 E\right) \varphi_{k} .
$$

\section{Acknowledgment.}

The authors thank Masaru Ikehata and David Isaacson for their valuable comments, and also the referee whose excellent advice brought a considerable improvement of the paper. SS was partly supported by Japan Society for the Promotion of Science (Grant-in-Aid for JSPS Fellows no. 00002757). GU was partly supported by NSF.

\section{Bibliography}

[1] Ammari, H.; Kwon, O.; Seo, J. K.; Woo, E. J. T-scan electrical impedance imaging system for anomaly detection. SIAM J. Appl. Math. 65 (2004), no. 1, 252-266.

[2] Ammari, H.; Uhlmann, G. Reconstruction of the potential from partial Cauchy data for the Schrödinger equation. Indiana Math. J. 53 (2004), 169-184.

[3] Andersen, K. E.; Brooks, S. P.; Hansen, M. B. A Bayesian approach to crack detection in electrically conducting media. Inverse Problems 17 (2001), 121-136.

[4] Astala, K.; Päivärinta, L. Calderón's inverse conductivity problem in the plane. Ann. of Math. 163 (2006), 265-299.

[5] Bal, G. Reconstructions in impedance and optical tomography with singular interfaces. Inverse Problems 21 (2005), 113-131.

[6] Baratchart, L.; Abda, A. B.; Hassen, F. B.; Leblond, J. Recovery of pointwise sources or small inclusions in 2D domains and rational approximation. Inverse Problems 21 (2005), 51-74.

[7] Blue, R. S.; Isaacson, D.; Newell, J. C. Real-time three-dimensional electrical impedance imaging. Physiol. Meas. 21 (2000), 15-26.

[8] Borcea, L. Electrical impedance tomography. Inverse Problems 18 (2002), R99-R136. Addendum, Inverse Problems 19 (2003), 997-998.

[9] Brühl, M. Explicit characterization of inclusions in electrical impedance tomography. SIAM J. Math. Anal. 32 (2001), 1327-1341. 
[10] Brühl, M.; Hanke, M. Numerical implementation of two noniterative methods for locating inclusions by impedance tomography. Inverse Problems 16 (2000), 1029-1042.

[11] Brühl, M.; Hanke, M.; Vogelius, M. S. A direct impedance tomography algorithm for locating small inhomogeneities. Numer. Math. 93 (2003), 635-654.

[12] Calderón, A. P. On an inverse boundary value problem. Seminar on numerical analysis and its applications to continuum physics (Soc. Brasileira de Matemàtica 1980), 65-73.

[13] Cedio-Fengya, D. J.; Moskow, S.; Vogelius, M. S. Identification of conductivity imperfections of small diameter by boundary measurements. Continuous dependence and computational reconstruction. Inverse Problems 14 (1998), 553-595.

[14] Cheney, M.; Isaacson, D.; Newell, J. C. Electrical Impedance Tomography. SIAM Review 41 (1999), no. 1, 85-101.

[15] Erhard, K.; Potthast, R. The point source method for reconstructing an inclusion from boundary measurements in electrical impedance tomography and acoustic scattering. Inverse Problems 19 (2003), 1139-1157.

[16] Friedman, A.; Isakov, V. On the uniqueness in the inverse conductivity problem with one measurement. Indiana Univ. Math. J. 38 (1989), 563-579.

[17] Hanke, M.; Brühl, M. Recent progress in electrical impedance tomography. Inverse Problems 19 (2003), S65-S90.

[18] Huang, C.; Lee, J.; Schultz, W. W.; Ceccio, S. L. Singularity image method for electrical impedance tomography of bubbly flows. Inverse Problems 19 (2003), 919-931.

[19] Hyvönen, N. Complete electrode model of electrical impedance tomography: approximation properties and characterization of inclusions. SIAM J. Appl. Math. 64 (2004), 902-931.

[20] Ikehata, M. Size estimation of inclusion. J. Inv. Ill-Posed Problems 6 (1998), no. 2, 127-140.

[21] Ikehata, M. Reconstruction of the shape of the inclusion from boundary measurements. Comm. Partial Differential Equations 23 (1998), 1459-1474.

[22] Ikehata, M. Enclosing a polygonal cavity in a two-dimensional bounded domain from Cauchy data. Inverse Problems 15 (1999), 1231-1241.

[23] Ikehata, M. Reconstruction of the support function for inclusion from boundary measurements. Journal of Inverse and Ill-posed Problems 8 (2000), 367-378.

[24] Ikehata, M. On reconstruction in the inverse conductivity problem with one measurement. Inverse Problems 16 (2000), 785-793.

[25] Ikehata, M. On reconstruction from a partial knowledge of the Neumann-to-Dirichlet operator. Inverse Problems 17 (2001), 45-51.

[26] Ikehata, M. Reconstruction of inclusion from boundary measurements. J. Inv. Ill-Posed Problems 10 (2002), 37-65.

[27] Ikehata, M. Mittag-Leffler's function and extracting from Cauchy data. Contemporary Mathematics (Inverse Problems and Spectral Theory, ed. H. Isozaki) 348 (2004), 41-52.

[28] Ikehata, M.; Ohe, T. A numerical method for finding the convex hull of polygonal cavities using the enclosure method. Inverse Problems 18 (2002), 111-124.

[29] Ikehata, M.; Siltanen, S. Numerical method for finding the convex hull of an inclusion in conductivity from boundary measurements. Inverse Problems 16 (2000), 1043-1052.

[30] Ikehata, M.; Siltanen, S. Electrical impedance tomography and Mittag-Leffler's function. Inverse Problems 20 (2004), 1325-1348.

[31] Isakov, V. On uniqueness of recovery of a discontinuous conductivity coefficient. Comm. Pure Appl. Math. 41 (1988), 865-877.

[32] Isozaki, H. The $\bar{\partial}$-theory for inverse problems associated with Schrödinger operators on hyperbolic spaces. Publ. RIMS, Kyoto Univ., in press.

[33] Isozaki, H.; Uhlmann, G. Hyperbolic geometry and the local Dirichlet-to-Neumann map. Advances in Math. 188 (2004), 294-314. 
[34] Ito, K.; Kunisch K.; Li, Z. Level-set function approach to an inverse interface problem. Inverse Problems 17 (2001), 1225-1242.

[35] Jonsson, E. Approximate Dirichlet to Neumann maps used in the partial reconstruction of conductivity distributions. Doctoral dissertation, Rensselaer Polytechnic Institute, 1997.

[36] Jordana, J.; Gasulla, M.; Pallas-Areny, R. Electrical resistance tomography to detect leaks from buried pipes. Meas. Sci. Technol. 12 (2001), 1061-1068.

[37] Jossinet J. The impedivity of freshly excised human breast tissue. Physiol. Meas. 19 (1998), 61-75.

[38] Kaipio, J. P.; Kolehmainen, V.; Somersalo, E.; Vauhkonen, M. Statistical inversion and Monte Carlo sampling methods in electrical impedance tomography. Inverse Problems 16 (2000), $1487-1522$.

[39] Kang, H.; Seo, J. K. The inverse conductivity problem with one measurement: uniqueness of balls in $\mathbb{R}^{3}$. SIAM J. Appl. Math. 59 (1999), 1533-1539.

[40] Kang, H.; Seo, J. K.; Sheen, D. The inverse conductivity problem with one measurement: stability and estimation of size. SIAM J. Math. Anal. 28 (1997), 1389-1405.

[41] Kenig, C.; Sjöstrand, J.; Uhlmann, G. The Calderón problem with partial data. Ann. of Math., in press.

[42] Kohn, R. V.; Vogelius, M. Determining conductivity by boundary measurements II. Interior results. Comm. Pure Appl. Math. 38 (1985), 643-667.

[43] Kwon, O.; Seo, J. K. Total size estimation and identification of multiple anomalies in the inverse conductivity problem. Inverse Problems 17 (2001), 59-75.

[44] Kwon, O.; Seo, J. K.; Yoon, J. R. A real-time algorithm for the location search of discontinuous conductivities with one measurement. Comm. Pure Appl. Math. 55 (2002), 1-29.

[45] Kwon, O.; Yoon, J. R.; Seo, J. K.; Woo, E. J.; Cho, Y. G. Estimation of anomaly location and size using electrical impedance tomography. IEEE Trans. Biomedical Engineering 50 (2003), 89-96.

[46] Lebedev, N. N. Special Functions and Their Applications, Dover, New York, 1972.

[47] Metherall, P.; Smallwood, R. H.; Barber, D. C. Three dimensional electrical impedance tomography of the human thorax. 18th annual international conference of the IEEE Engineering in Medicine and Biology Society (Amsterdam, 1996).

[48] Mueller, J. L.; Isaacson, D.; Newell, J. C. A Reconstruction Algorithm for Electrical Impedance Tomography Data Collected on Rectangular Electrode Arrays. IEEE Trans. Biomedical Engineering 46 (1999), 1379-1386.

[49] Nachman, A. I. Reconstruction from boundary measurements. Ann. of Math. 128 (1988), 531576.

[50] Nachman, A. I. Global uniqueness for a two dimensional inverse boundary value problem. Ann. of Math. 143 (1996), 71-96.

[51] Nakamura, G.; Siltanen, S.; Tanuma, K.; Wang, S. Numerical recovery of conductivity at the boundary from the localized Dirichlet to Neumann map. Computing 75 (2005), 197-213.

[52] Novikov, R. G. A multidimensional inverse spectral problem for the equation $-\Delta \psi+(v(x)-$ $E u(x)) \psi=0$. Funktsional. Anal. i Prilozhen. 22 (1988), no. 4, 11-22. Translation in Funct. Anal. Appl. 22 (1989), no. 4, 263-272.

[53] Ramirez, A.; Daily, W.; LaBrecque, D.; Owen, E.; Chesnut, D. Monitoring an underground steam injection process using electrical resistance tomography. Water Resources Research 29 (1993), 73-87.

[54] Ramirez, A.; Daily, W.; Binley, A.; LaBrecque, D.; Roelant, D. Detection of leaks in underground storage tanks using electrical resistance methods. J. Envir. Eng. Geophys. 1 (1996), 189-203. 
[55] Rondi, L.; Santosa, F. Enhanced electrical impedance tomography via the Mumford-Shah functional. Control, Optimisation, and Calculus of Variations 6 (2001), 517-538.

[56] Slater, L.; Binley, A. M.; Daily, W.; Johnson, R. Cross-hole electrical imaging of a controlled saline tracer injection. Journal of Applied Geophysics 44 (2000), 85-102.

[57] Sylvester, J.; Uhlmann, G. A global uniqueness for an inverse boundary value problem. Ann. of Math. 125 (1987), 153-169.

[58] Vauhkonen, P. Image Reconstruction in Three-Dimensional Electrical Impedance Tomography. Doctoral dissertation, University of Kuopio, 2004.

[59] Zou, Y.; Guo, Z. A review of electrical impedance techniques for breast cancer detection, Med. Eng. Phys. 25 (2003), pp. 79-90.

Received Month 200X. 